\title{
Instagram Influencer Selection Analysis on the SME's Brand Campaign in Indonesia using Correlation Analysis (Case Study: IAM.ID)
}

\author{
Icha Isana Febriana, Creative and Cultural Entrepreneurship, Master of Business Management, Institute Teknologi \\ Bandung, Bandung, Indonesia. Email- icha-isanafebriana@sbm-itb.ac.id
}

Isti Raafaldini Mirzanti, Entrepreneurship and Technology Management, Institut Teknologi Bandung, Bandung, Indonesia. Email-isti@sbm-itb.ac.id

\begin{abstract}
The number of Small Medium Enterprises (SMEs) in Indonesia reached 64,194,057 units in 2018 and made a good impact on Indonesia's economy. However, it turns out that SMEs face various problems, such as business strategy, marketing, and branding. Only 12\% of SMEs currently use digital as one of their platforms and most of these businesses are still in stagnant condition. Currently, SMEs are starting to use influencers as their marketing strategy on Instagram. However, it is difficult to find a suitable influencer for their product or service due to so many influencers with various categories. The market potential in this field is quite high considering that Indonesia has the largest number of social media users in the world, especially Facebook and Instagram. Globally 5-10 billion USD marketing is done through influencer marketing in 2020, 65\% of companies plan to use more influencer marketing and $92 \%$ of customers trust personal recommendations more than conventional advertising. An analysis was conducted of the selection of Instagram influencers on the brand campaign in Indonesia through a case study from IAM.ID. This company provides solutions in the fields of influencer marketing and technology with influencers data analytics. IAM.ID services include content creations, endorsement, store visits, and influencer's community. In this research, the hypothesis is based on the level of engagement and influencer category. Influencers are considered more effective if they match the advertised brand. Influencer data analytics are obtained from engagement rate (ER) influencers, the number of followers, influencer categories, and content analysis. Brand analysis is obtained from brand preferences obtained through questionnaires. Then a correlation analysis was carried out on the ER campaign and the result was that both the number of followers and ER influencers had a strong relationship to the ER campaign. Besides, the suitability of the influencer category can also be checked using content analysis.
\end{abstract}

Keywords:

SMEs, influencer, engagement-rate, influencer data analytics, correlation analysis, content analysis

Article Received: 18 October 2020, Revised: 3 November 2020, Accepted: 24 December 2020

\section{Introduction}

The number of Small Medium Enterprises (SMEs) in Indonesia reached 64,194,057 units in 2018 (Indonesia Ministry of Cooperatives and Small and Medium Enterprises, 2019). This is predicted to continue to increase in the year ahead and will have a good impact on the economy in Indonesia. However, it turns out that SMEs face various problems, such as business strategy, marketing, and branding. Only $12 \%$ of SMEs currently use digital as one of their platforms and these businesses are mostly at stagnant condition. This is because SMEs do not know how to grow a business and lack knowledge about good business management. Many SMEs only focus on producing goods without thinking about how to grow their business. As a result, SMEs find it difficult to increase their business level.

Meanwhile, the digital era has the potential to change every aspect of everyday life. It will reshape the way people create decisions, improve customer experience and create new business models. The number of internet users in Indonesia currently reaches 175.4 million users, an increase of $17 \%$ (25 million users) compared to the previous year. This means that approximately $64.8 \%$ of Indonesian people have been able to use the internet and 150 million are active users of social media [1]. This is good news for SMEs to move ahead, especially on the online marketing platform. There are a lot of ways to connect with an audience online, such as social media platform, 
influencer marketing, email marketing, content marketing, Search Engine Optimization (SEO) and Pay-per-click (PPC). The focus of this research will be influencer marketing with engagement rate as its main parameter. Globally 5-10 billion USD marketing is done through influencer marketing in 2020, $65 \%$ of companies plan to use more influencer marketing and $92 \%$ of customers trust personal recommendations more than conventional advertising [2].

An influencer is someone who has large followers so they have a better chance to promote a product through their social media content. There are 4 (four) influencer categories based on the number of followers, namely nano (1,000-10,000 followers), micro (10,000-100,000 followers), macro (100,000-1,000,000 followers), and mega (above 1,000,000 followers) influencers. Although nano and micro influencers have fewer followers than other types of influencers, they have interests in specific fields, such as beauty, business, automotive, health, parenting, traveling, technology, culinary, and so on. The advantages of nano and micro influencers are the high engagement-rate and trust from their followers.

Influencer marketing is a new style in the world of marketing. The magnitude of a "character's influence" is the reason why influencer marketing has become one of the choices in the marketing world. According to Nielsen [3], 92\% of consumers trust recommendations from someone more than through conventional advertising. However, this opportunity has not been maximized by SMEs in Indonesia due to a lack of knowledge and budgets. Influencer marketing has been described as a type of native advertising, branded entertainment, or highly credible electronic word of mouth because the commercial posts usually are daily routine narratives that SMIs share with their followers [4]. There are many ways to analyze a campaign using influencers. One way is through measuring their engagement rate. Engagement rate is a measure that detects how actively your content engages with your audience. Interaction can be done via the "like" button, comment, save, or share on social media. It is a measure most often used in analyzing the effectiveness of a brand's campaign. The rest of the paper is organized as follows. The business issue will be explained in section II. Experimental results are presented in section III. Concluding remarks are given in section IV.

\section{Business Issue}

In this research, a hypothesis has been made which is the behavior of brand choosing influencers is by their engagement rate (percentage of interaction in the form of comments and likes) and the influencer category (niche or content category). It is said that the influencers are more effective if they match the advertised brand.

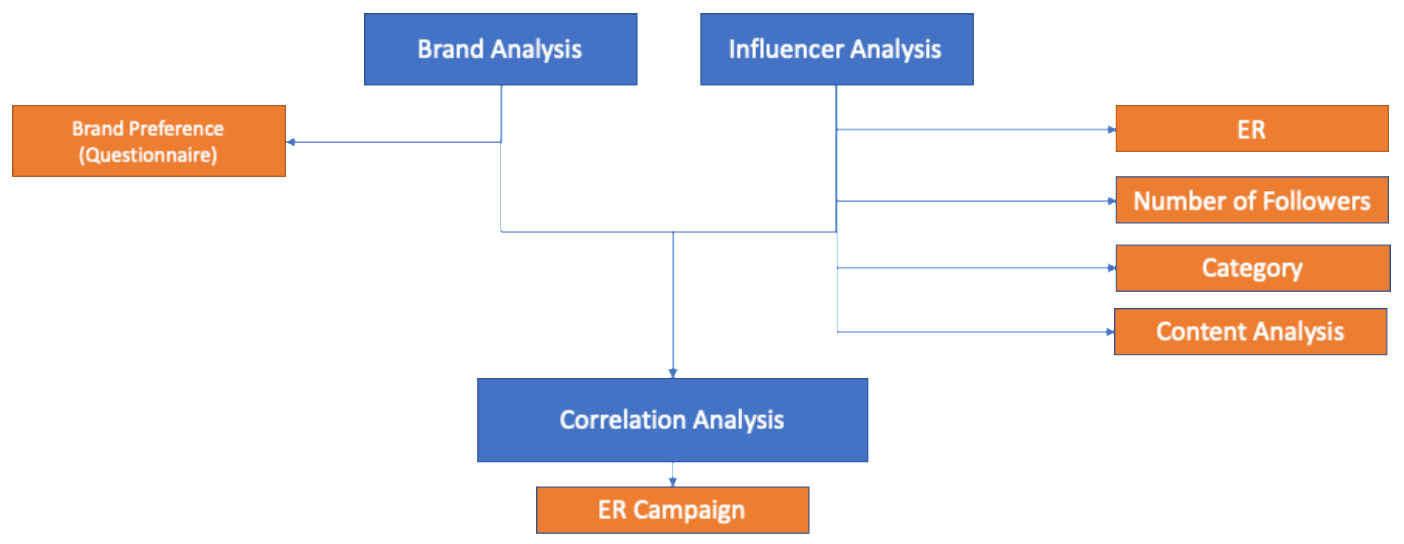

Figure 1 Conceptual Framework

The influencer analysis will be consist of influencer ER, number of followers, influencer category, and content analysis. Then it will be analyzed with correlation analysis to see how it 
relates to the ER campaign. The brand analysis gained from customer feedback that already used IAM.ID service as brand preference. Then analyzed as additional data for correlation analysis. Besides, the suitability of the influencer category can also be checked using content analysis.

\section{Experiment and Result \\ 3.1 Correlation Analysis -}

Data collected from the campaign results on the IAM.ID system. This data was taken from 13 brand campaigns involving 42 influencers in two categories. The campaign category used is the food category and the fashion \& beauty category. The data collected consisted of influencer data and campaign data. For influencer data, the data collected includes influencer username, influencer category, number of followers, influencer ER, and influencer caption data. As for the data from the campaign results, the data collected is the number of likes, comments, and shares.

The main objective of the analysis carried out is to determine the significance of the number of followers and ER Influencers on the ER of a campaign carried out by a brand in two campaign categories, namely the campaign in the food category and the campaign in the beauty \& fashion category. Outlier analysis is used to detect data that stands out compared to other data. This detection process is used to reduce errors and the correlation that will be carried out. This outlier analysis was carried out using Microsoft Excel's graphs.

The tools used to perform correlation analysis are using the trendline feature by Excel's in determining the coefficient of determination ( $R$ Square). $\mathrm{R}$ determination explains how far the dependent data (in this case the ER campaign) can be explained by independent data (in this case the number of influencer followers or the number of ER influencers). This correlation analysis uses data in the table that has eliminated outliers. The following is a rule of thumb based on Moore, et.al. [5], to interpret the strength of the relationship based on R Square:
- if R-squared value $<0.3$ this value is generally considered a none or very weak effect size,

- if R-squared value $0.3<\mathrm{r}<0.5$ this value is generally considered a weak or low effect size,

- if R-squared value $0.5<\mathrm{r}<0.7$ this value is generally considered a moderate effect size,

- if R-squared value $r>0.7$ this value is generally considered strong effect size.

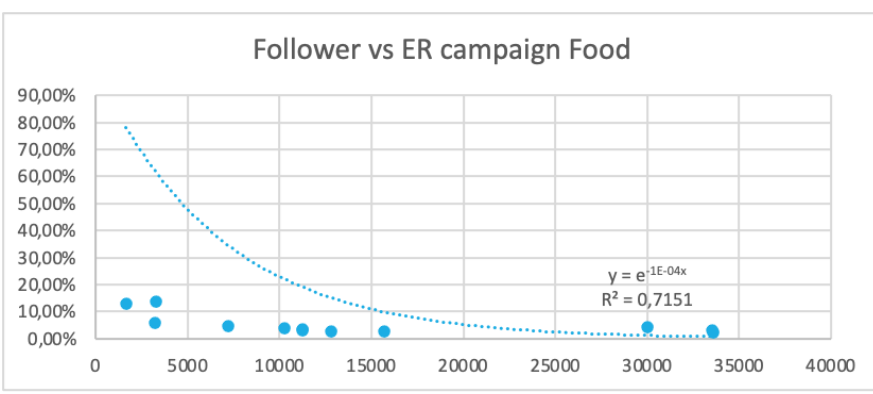

Figure 2 Correlation Analysis of Number of Follower and ER Campaign in Food Category

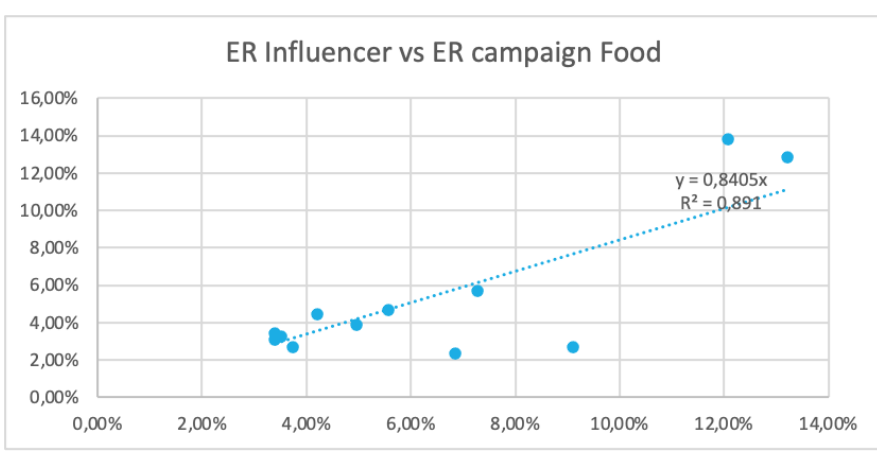

Figure 3 Correlation Analysis of ER Influencer and ER Campaign in Food Category

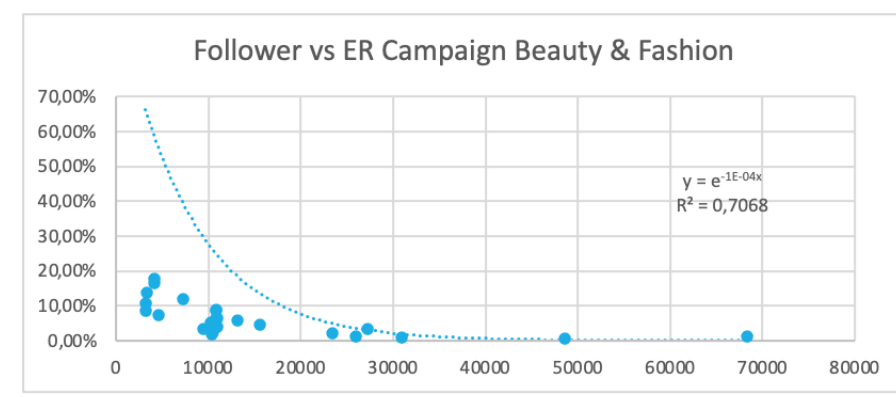

Figure 4 Correlation Analysis of Number of Follower and ER Campaign in Beauty \& Fashion Category 


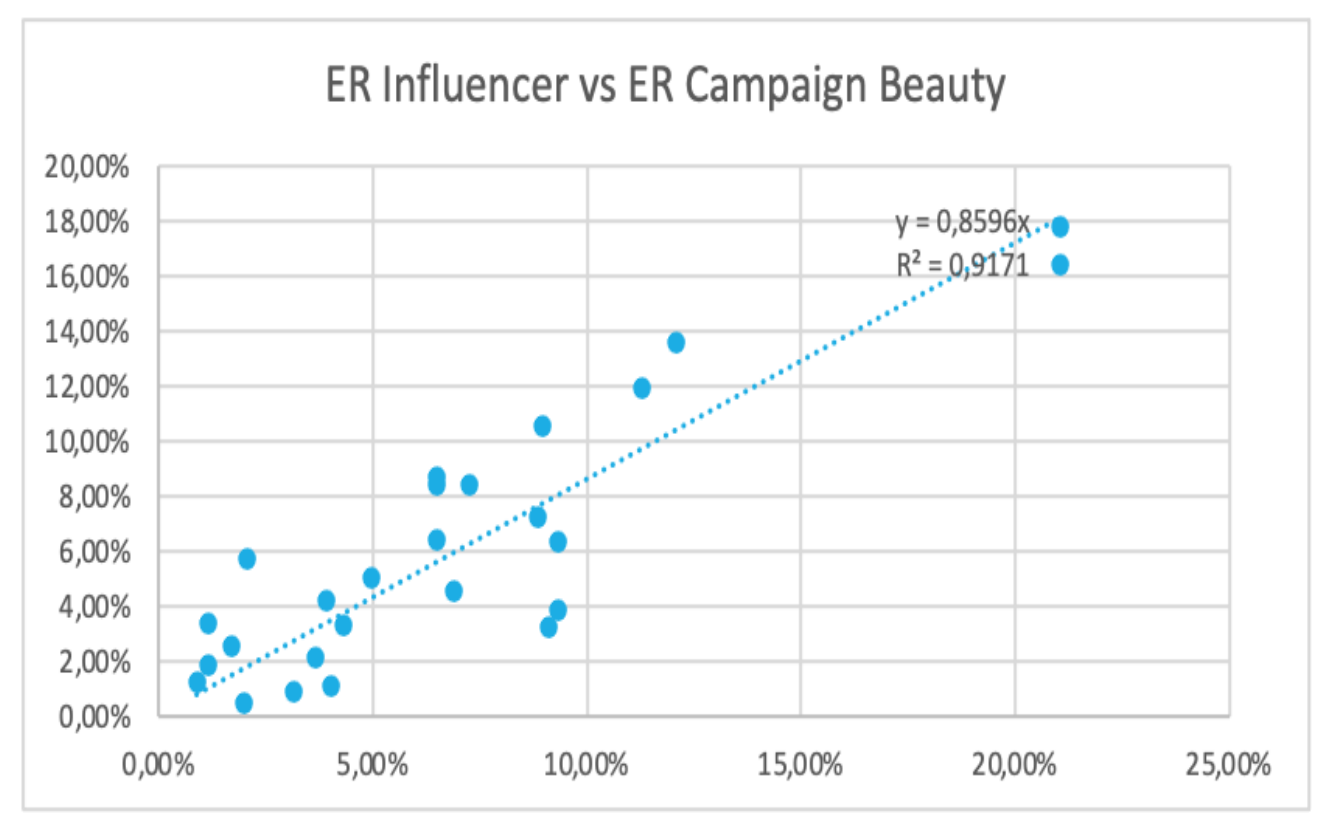

Figure 5 Correlation Analysis of ER Influencer and ER Campaign in Beauty \& Fashion Category

To find out whether there is a significant difference if the data from the two categories are combined we perform an analysis for the combined category.

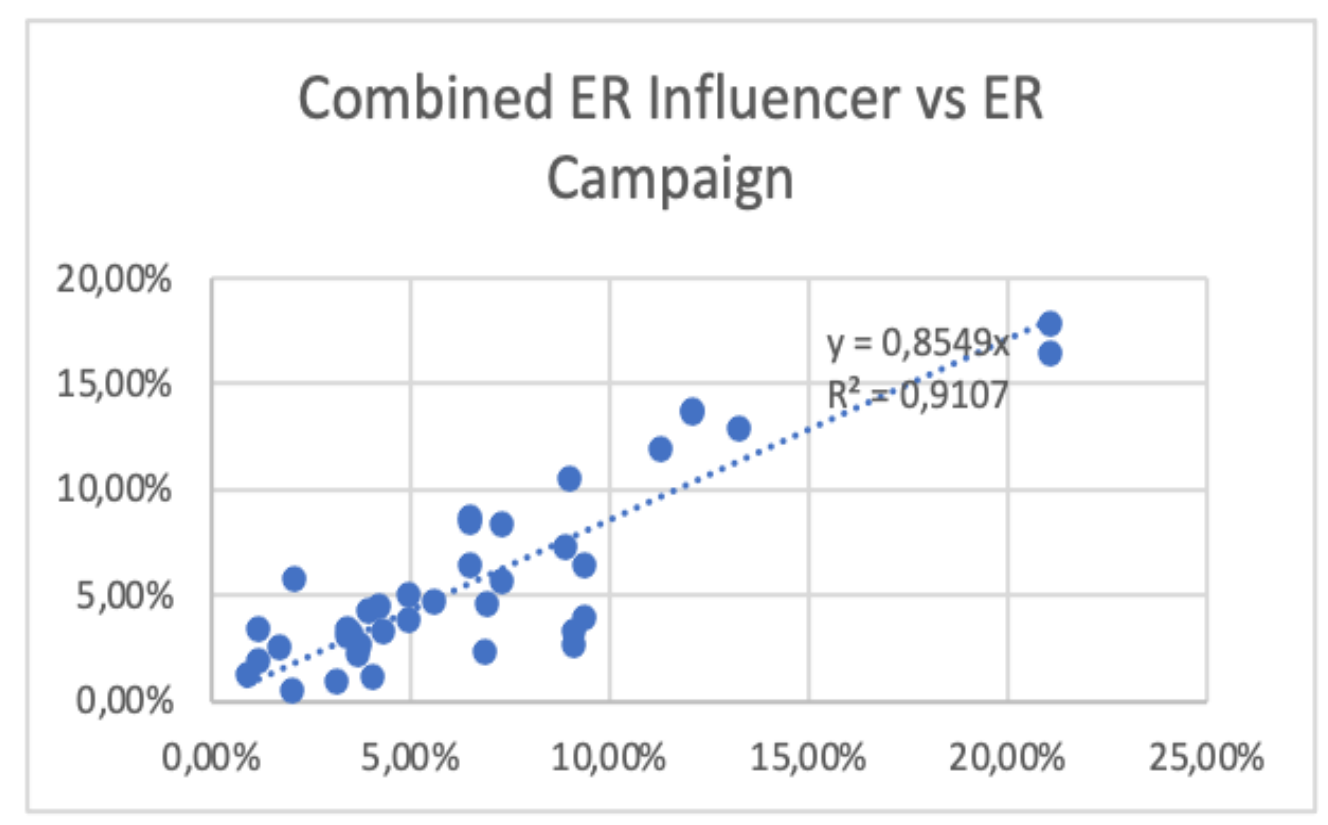

Figure 6 Correlation Analysis of ER Influencer \& Combined ER Campaign

\subsection{Content Analysis -}

This content analysis aims to analyze the correlation between the effect of the suitability of captions (text or sentences that are posted) that are often used by influencers on the ER campaign. This caption data is taken from the last 30 influencers post data. From 42 influencer data collected, 27 influencers were successfully obtained captions. To see if an influencer fits into the campaign category, we checked the 50 words most frequently used by each influencer. Table 1 shows the results of measuring the percentage of influencer content conformity to the campaign category. 
Table 1 Content Analysis Data

\begin{tabular}{|c|c|c|c|c|c|c|c|}
\hline No & Campaign Category & Username & $\begin{array}{c}\text { Match } \\
\text { Percentage }\end{array}$ & ER Campaign & $\begin{array}{c}\text { Total } \\
\text { Keyword } \\
\text { Weight }\end{array}$ & $\begin{array}{c}\text { Match } \\
\text { Keyword }\end{array}$ & $\begin{array}{c}\text { Match } \\
\text { Keyword } \\
\text { Weight }\end{array}$ \\
\hline 1 & \multirow{3}{*}{ Beautfy \& Fashion } & aliyaashf & $44 \%$ & $3,38 \%$ & 117 & 21 & 52 \\
\hline 2 & & ifahh26 & $60 \%$ & $10,55 \%$ & 104 & 24 & 62 \\
\hline 3 & & kasyaaptr & $56 \%$ & $17,83 \%$ & 16 & 9 & 9 \\
\hline 4 & \multirow{3}{*}{ Beautfy \& Fashion } & virabs & $50 \%$ & $0,92 \%$ & 26 & 10 & 13 \\
\hline 5 & & nindiayg & $51 \%$ & $0,52 \%$ & 76 & 25 & 39 \\
\hline 6 & & dellaaktialema & $48 \%$ & $8,41 \%$ & 60 & 22 & 29 \\
\hline 7 & \multirow{4}{*}{ Beautfy \& Fashion } & icacsss & $70 \%$ & $8,70 \%$ & 82 & 31 & 57 \\
\hline 8 & & rakaswara_vyatrantra & $26 \%$ & $1,24 \%$ & 127 & 16 & 33 \\
\hline 9 & & ghinaafaa & $15 \%$ & $3,90 \%$ & 144 & 9 & 22 \\
\hline 10 & & novinurul_ummusha & $63 \%$ & $14,98 \%$ & 134 & 30 & 84 \\
\hline 11 & \multirow{3}{*}{ Beautfy \& Fashion } & dheadeil & $40 \%$ & $3,28 \%$ & 83 & 19 & 33 \\
\hline 12 & & shevinasabila & $32 \%$ & $13,58 \%$ & 72 & 15 & 23 \\
\hline 13 & & fe_brinaaptr & $30 \%$ & $13,78 \%$ & 120 & 11 & 36 \\
\hline 14 & \multirow{3}{*}{ Food } & fidiantif & $24 \%$ & $3,40 \%$ & 55 & 10 & 13 \\
\hline 15 & & meulatipucuk_ & $8 \%$ & $2,70 \%$ & 131 & 4 & 10 \\
\hline 16 & & anggitaeget & $19 \%$ & $3,23 \%$ & 130 & 10 & 25 \\
\hline 17 & \multirow{4}{*}{ Food } & Haninazhr & $14 \%$ & $2,71 \%$ & 65 & 6 & 9 \\
\hline 18 & & nendenhnf & & $61,03 \%$ & 54 & 15 & 22 \\
\hline 19 & & desiipermaataa & $5 \%$ & $2,36 \%$ & 119 & 1 & 6 \\
\hline 20 & & jonirizkip_. & $5 \%$ & $4,44 \%$ & 85 & 3 & 4 \\
\hline 21 & Food & vilkanindyandes & $6 \%$ & $4,68 \%$ & 67 & 2 & 4 \\
\hline 22 & \multirow{2}{*}{ Beauty \& Fashion } & elviraaminitiii & $19 \%$ & $1,15 \%$ & 54 & 7 & 10 \\
\hline 23 & & putiinilamsari & $59 \%$ & $4,25 \%$ & 136 & 25 & 80 \\
\hline 24 & Beauty \& Fashion & drandrenaa & $81 \%$ & $11,93 \%$ & 321 & 37 & 259 \\
\hline 25 & Beauty \& Fashion & tyasirene & $32 \%$ & $7,26 \%$ & 96 & 10 & 31 \\
\hline 26 & \multirow{2}{*}{ Beauty \& Fashion } & amellamnda & $0 \%$ & $2,19 \%$ & 31 & 1 & 0 \\
\hline 27 & & ervia_viaa & $13 \%$ & $5,73 \%$ & 23 & 3 & 3 \\
\hline
\end{tabular}

\section{Match Percentage vs ER Campaign}

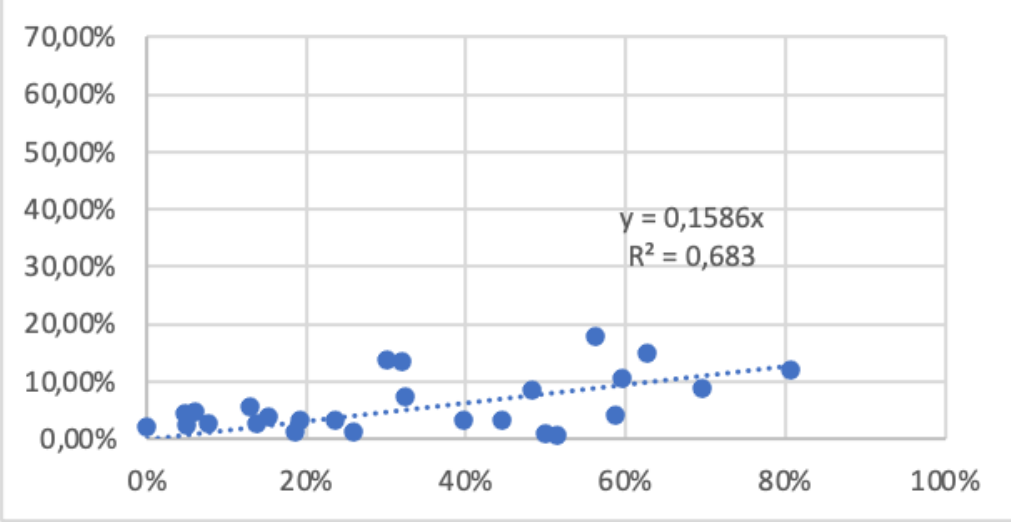

Figure 7 Match Percentage vs ER Campaign

\subsection{Brand Preference Analysis}

As much as $12.44 \%$ of the total number of IAM.ID customers, which is 28 respondents (current total IAM.ID client is 225 SMEs) involved in this research. The product type comes from 4 categories, which are fashion, beauty, food, and others. Of the 28 respondents, $57.1 \%$ or 16 respondents felt that marketing through influencers was effective. There are still some opinions that the campaign is ineffective because there are no direct sales for the product right after the campaign is held. From data, it is known that $28.6 \%$ or as many as 8 brands received direct sales after the campaign and 20 others did not get direct sales. This can be further analyzed both in terms of the use of influencer types (nano, micro, or macro influencers) and the category of the influencers themselves The survey results show that the 4 main parameters of how SMEs choosing influencers are:
1)
Number of followers
2) Influencer profile
3) Influencer content
4) Engagement rate.

From the result, $96.4 \%$ or 27 brands will return to using IAM services, and also it can be seen that 
endorsement has a better effect than paid promotion especially for nano and micro influencers From the results of the campaign conducted by IAM.ID, an endorsement is more effective for nano or micro influencers considering that authentic reviews are preferred by their followers so that the tendency for followers to buy or try these products is much higher than paid promotion. About $53.6 \%$ of respondents or 15 respondents stated that macro influencers were the most effective influencers in conducting a campaign, then followed by micro influencers, nano influencers, and mega influencers. The results show that the reason why using an influencer is effective for their brand are because:

1) nano and micro influencers

2) Many followers of these influencers know about the products or services offered by these SMEs even though they don't buy them

3) High influencer engagement rates have a better effect on campaign results

4) The cost is very much more affordable for the use of nano and micro influencers because SMEs have limited budgets

5)

Have better interactions on SMEs owner's Instagram after the campaign

6)

SME owners are aware of the trust and emotional closeness generated by nano, micro and macro influencers.

7) The use of macro influencers has a more significant effect in increasing the number of followers of Instagram for SMEs owners.

From all results analysis that has been carried out, several alternative solutions can be given as follows:

1)

In the selection of influencers, ER consideration is an appropriate consideration considering the results of the analysis show that ER has a good correlation with the success of a brand with the assumption that SME chooses influencers with the same interests as the brand category, for example, fashion brands will choose fashion influencers.

2) The higher the number of followers correlates with a decrease in ER. If SME wants to increase brand awareness with the aim of a broad reach, it is better if you use a macro influencer or mega influencer even though it does not have a better ER compared to nano influencers and micro influencers.

\section{CONCLUSION}

The business issue discussed in this research is to assess and optimize the influencer selection and how the influence of content or influencer category on brand interests in choosing them. Engagement rate is a very important component of Instagram influencer selection for the campaign to be successful. Categories related to engagement rate are brand and influencer fit, the right target market, honest language, and passion for the product. For this reason, influencer creativity also determines the success of an ongoing campaign.

Based on the correlation analysis that has been made, the results obtained from the food campaign show a strong relationship between the number of followers to the ER campaign. So it can be stated that the higher the number of followers, the ER campaign will decrease exponentially. It is also known that the ER influencer relationship with ER campaigns has a strong relationship as well, the higher the ER influencer and the ER campaign will increase linearly. It is no different from fashion and beauty campaigns that also has the same result. When the two data are combined, it doesn't affect the result that the bigger the follower, the ER campaign will decrease exponentially and the higher the ER influencer, the ER campaign will increase linearly. This shows that the relationship between the number of followers or ER influencers to the ER campaign is generally accepted for all influencer categories.

From the analysis, it can be concluded that: 
1. ER Campaign is directly proportional to ER Influencer, the higher the ER influencer, the higher the ER campaign.

2. The model of the relationship between the number of followers and the ER campaign is to follow the exponential model, the higher the number of followers the ER campaign tends to decrease.

3. According to the initial hypothesis that to choose an influencer for a higher ER campaign, SME should choose one with a high ER.

4. If the purpose of the campaign is to increase SME's social media engagement, then it is more suitable to choose a micro influencer or one with a smaller number of followers because in fact, the higher the number of followers, the lower the ER will be.

From the results obtained through brand preference, it is known that endorsements have a better success rate than paid-promotes for nanoscale and micro influencers. This because followers trust the product if influencers also personally review their experience when using the product compared to only posting advertisement content in the form of images or videos offered by paid-promote. From brand preference, it is also known that the success of the campaign can be seen if the brand matches the influencer category. The increase of influencer's ER will be correlated with the increase of ER campaign linearly, where a higher number of followers will tend the ER campaign to decrease exponentially. It also concluded that choosing influencers with higher ER will have a result in a higher ER campaign. If SME has a purpose to increase its social media engagement, then it is more suitable to choose a micro influencer or nano influencers because the higher the number of followers, the lower the ER. From this research, it is known that the ER campaign can be predicted from ER influencers or the number of followers. Also, determining the influencer category can also be done by using content analysis. Generally, influencers will determine what content they like or what content represents their personality on Instagram, for example, food influencers, fashion influencers, beauty influencers, and so on. However, this also needs to be checked again for its suitability with real data on Instagram feed using content analysis. With this content analysis, it can be tested whether the influencer category matches before running the campaign.

\section{REFERENCES}

[1] Statista Research Department, 2020, Internet Usage in Indonesia - Statistics and Facts, October 14, Viewed 27 October 2020 at https://www.statista.com/topics/2431/internetusage-in-indonesia.

[2] Bailis, R., The State of Influencer Marketing: 10 Influencer Marketing Statistics to Inform Where You Invest, Viewed 29 October 2020 at https://www.bigcommerce.com/blog/influence r-marketing-statistics/\#what-is-influencermarketing.

[3] DeVeirman, M., Cauberghe, V., \& Hudders, L., 2017, Marketing through Instagram Influencers: The Impact of Number of Followers and Product Divergence on Brand Attitude, International Journal of Advertising 36, $5: 798-828$.

[4] Nielsen, 2012, Consumer Trust in Online, Social, and Mobile Advertising Grows, 4 November. Viewed 12 January 2021 at https://www.nielsen.com/us/en/insights/article /2012/consumer-trust-in-online-social-andmobile-advertising-grows/.

[5] Moore, D.S., Notz, W., Fligner, M.A., 2013, The Basic Practice of Statistics, New York : W.H., Freeman and Co. 